Check for updates

Cite this: Phys. Chem. Chem. Phys., 2020, 22, 6013

DOI: $10.1039 / \mathrm{d} 0 \mathrm{cp} 90056 \mathrm{~g}$

rsc.li/pccp

\section{Correction: Enhancement of electron accepting ability of para-benzoquinone by a single water molecule}

\author{
Golda Mensa-Bonsu, (D) Aude Lietard (D) and Jan R. R. Verlet (DD* \\ Correction for 'Enhancement of electron accepting ability of para-benzoquinone by a single water \\ molecule' by Golda Mensa-Bonsu et al., Phys. Chem. Chem. Phys., 2019, 21, 21689-21692.
}

We recently reported the observation of an enhanced electron accepting ability of para-benzoquinone (pBQ) when solvated by a single water molecule in the published paper. The main experimental evidence for this was taken from the frequency-resolved (2D) photoelectron (PE) spectra. Unfortunately, we have since discovered that the presented spectra were erroneous. Specifically, in analysis of the PE images using the polar onion peeling routine, ${ }^{1}$ we had omitted to take into account the Jacobian that is required

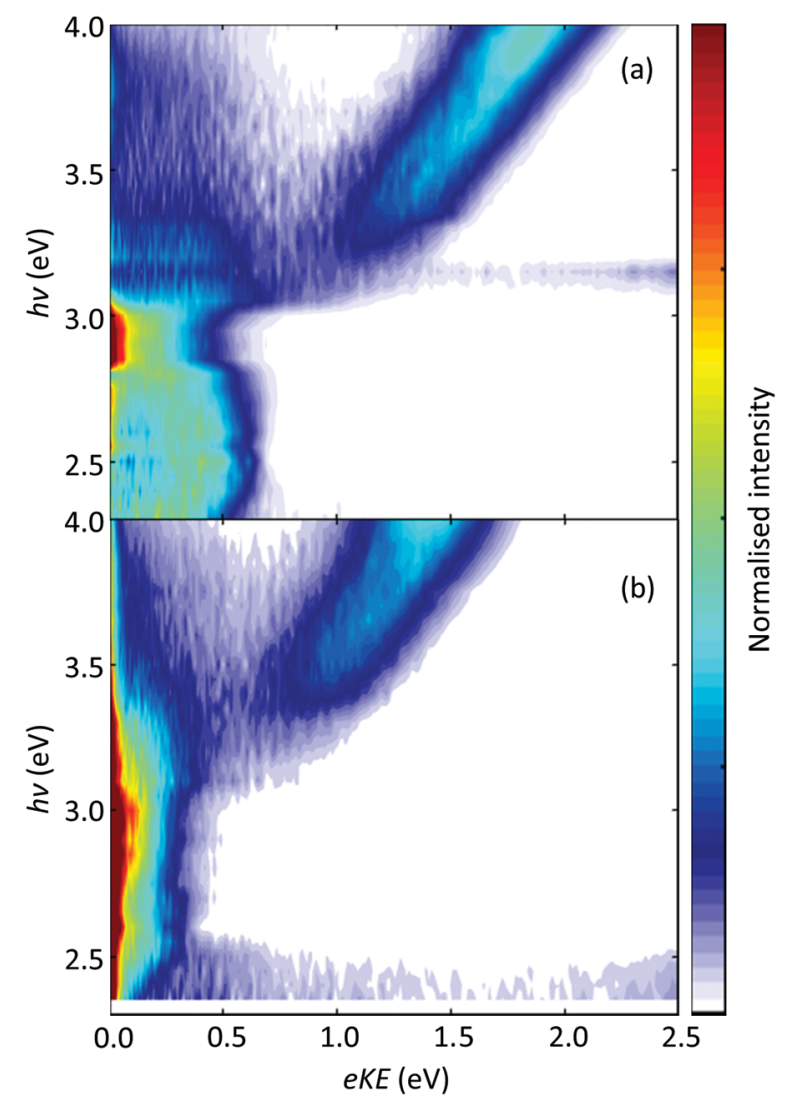

Fig. 2 Corrected frequency-resolved (2D) photoelectron (PE) spectra of jet-cooled (a) $\mathrm{pBQ}^{-}$and (b) $\mathrm{pBQ}^{-}\left(\mathrm{H}_{2} \mathrm{O}\right)$ recorded in the range $2.3<h \nu<4.0$ eV. Each PE spectrum has been normalised to its total signal intensity. 


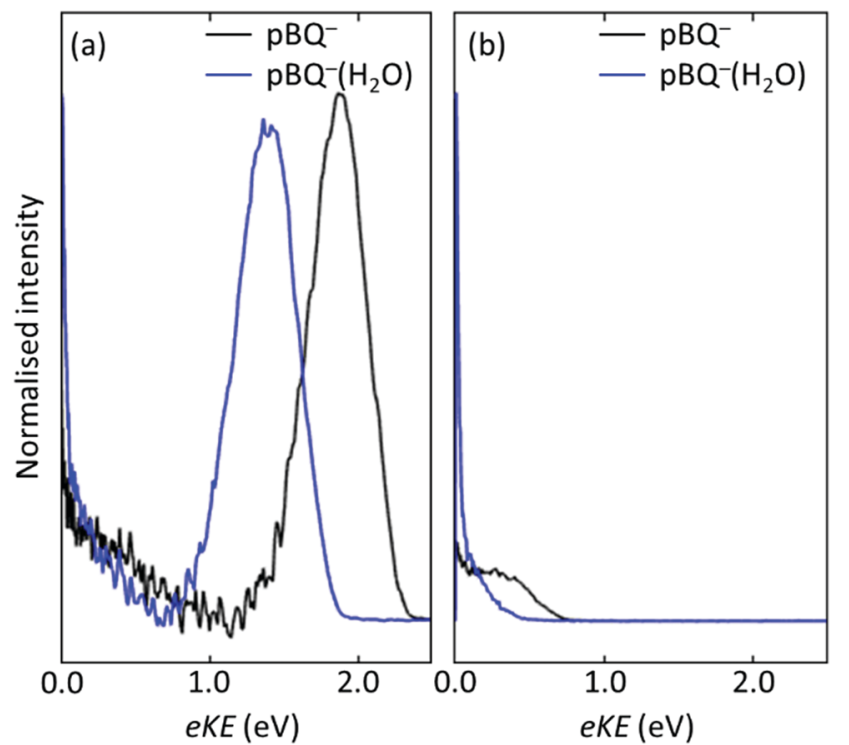

Fig. 3 Corrected photoelectron spectra measured at (a) $h \nu=4.00 \mathrm{eV}$ and (b) $h \nu=2.75 \mathrm{eV}$ for $\mathrm{pBQ}^{-}$(black lines) and $\mathrm{pBQ}{ }^{-}\left(\mathrm{H}_{2} \mathrm{O}\right)(\mathrm{blue}$ lines). Each $\mathrm{PE}$ spectrum has been normalised to its total signal intensity.

in the conversion from radius (velocity) to electron kinetic energy space. Hence, all the PE spectra presented require additional scaling by $1 / r$, where $r$ is the radius of the image collected in the velocity map imaging experiment.

The corrected results are shown in Fig. 2 and should replace those shown in Fig. 2 of the published article. Fig. 2(a) and (b) show the 2D PE spectra for $\mathrm{pBQ}^{-}$and $\mathrm{pBQ}^{-}\left(\mathrm{H}_{2} \mathrm{O}\right)$, respectively, taken over the range $2.3<\mathrm{eKE}<4.0 \mathrm{eV}$ in $0.05 \mathrm{eV}$ increments. The main conclusion of the previous study was that a single water molecule enhances the electron accepting ability of pBQ. This conclusion remains valid with the corrected data. The electron accepting ability is captured by the efficiency with which the ground electronic state is recovered when excited above the detachment threshold. The spectral signature of ground state recovery is the appearance of low-energy electrons. Fig. 2 clearly shows an overall enhancement of such low energy electrons over a broad range of photon energies and, hence, of the electron accepting ability in the presence of a water molecule. Fig. 3 shows the corrected 1D PE spectra, which should replace the erroneous Fig. 3 in the published article.

While the core conclusion is not affected by the correction, we also commented on the apparent discrepancy between the 2D PE spectra and a previous study from our group ${ }^{2,3}$ and we attributed the differences to the fact that the previous experiments were performed at $300 \mathrm{~K}$ instead of $10 \mathrm{~s}$ of $\mathrm{K}$ in the molecular beam experiments. Having corrected the erroneous $2 \mathrm{D}$ PE spectra of $\mathrm{pBQ}^{-}$, we now conclude that, in fact, the temperature has very little, if any, effect on the overall dynamics. Hence, any discussions offered to explain the differences are not relevant and should be disregarded.

The Royal Society of Chemistry apologises for these errors and any consequent inconvenience to authors and readers.

\section{Conflicts of interest}

There are no conflicts to declare.

\section{Acknowledgements}

This work was supported by the EPSRC (EP/R023085/1).

\section{References}

1 G. M. Roberts, J. L. Nixon, J. Lecointre, E. Wrede and J. R. R. Verlet, Rev. Sci. Instrum., 2009, 80, 053104.

2 D. A. Horke, Q. Li, L. Blancafort and J. R. R. Verlet, Nat. Chem., 2013, 5, 711-717.

3 C. W. West, J. N. Bull, E. Antonkov and J. R. R. Verlet, J. Phys. Chem. A, 2014, 118, 11346-11354. 